\title{
Administrative incidence of psychosis assessed in an early intervention service in England: first epidemiological evidence from a diverse, rural and urban setting
}

\author{
F. Cheng ${ }^{1,2,3}$, J. B. Kirkbride ${ }^{1,2}$, B. R. Lennox ${ }^{1,2}$, J. Perez ${ }^{3}$, K. Masson ${ }^{3}$, K. Lawrence ${ }^{3}$, K. Hill ${ }^{4}$, L. Feeley ${ }^{3}$, \\ M. Painter ${ }^{2}$, G. K. Murray ${ }^{1,2,3}$, O. Gallagher ${ }^{3,5}$, E. T. Bullmore ${ }^{1,2}$ and P. B. Jones ${ }^{1,2 *}$ \\ ${ }^{1}$ University Department of Psychiatry, University of Cambridge, UK \\ ${ }^{2}$ NIHR CLAHRC for Cambridgeshire and Peterborough, Douglas House, Cambridge, UK \\ ${ }^{3}$ CAMEO, Block 14, Ida Darwin, Fulbourn, Cambridge, UK \\ ${ }^{4}$ Norfolk and Waveney Mental Health NHS Foundation Trust, Hellesdon Hospital, Norwich, UK \\ ${ }^{5}$ NHS Greater Glasgow and Clyde, UK
}

Background. Early Intervention in Psychosis Services (EIS) for young people in England experiencing first-episode psychosis (FEP) were commissioned in 2002, based on an expected incidence of 15 cases per 100000 person-years, as reported by schizophrenia epidemiology in highly urban settings. Unconfirmed reports from EIS thereafter have suggested higher than anticipated rates. The aim of this study was to compare the observed with the expected incidence and delineate the clinical epidemiology of FEP using epidemiologically complete data from the CAMEO EIS, over a 6-year period in Cambridgeshire, for a mixed rural-urban population.

Method. A population-based study of FEP (ICD-10, F10-39) in people aged 17-35 years referred between 2002 and 2007; the denominator was estimated from mid-year census statistics. Sociodemographic variation was explored by Poisson regression. Crude and directly standardized rates (for age, sex and ethnicity) were compared with pre-EIS rates from two major epidemiological FEP studies conducted in urban English settings.

Results. A total of 285 cases met FEP diagnoses in CAMEO, yielding a crude incidence of 50 per 100000 personyears [95\% confidence interval (CI) 44.5-56.2]. Age- and sex-adjusted rates were raised for people from black ethnic groups compared with the white British [incidence rate ratio (IRR) 2.1, 95\% CI 1.1-3.8]. Rates in our EIS were comparable with pre-EIS rates observed in more urban areas after age, sex and ethnicity standardization.

Conclusions. Our findings suggest that the incidence observed in EIS is far higher than originally anticipated and is comparable to rates observed in more urban settings prior to the advent of EIS. Sociodemographic variation due to ethnicity and other factors extend beyond urban populations. Our results have implications for psychosis aetiology and service planning.

Received 17 February 2010; Revised 9 November 2010; Accepted 13 November 2010; First published online 23 December 2010

Key words: Early intervention in psychosis, epidemiology, health services research, incidence, psychotic disorders, public health.

\section{Introduction}

Much of our knowledge about the clinical epidemiology of psychotic disorders comes from studies based in predominately urban settings, often cities (March et al. 2008), and predicated on health service models

\footnotetext{
* Address for correspondence: Professor P. B. Jones, University Department of Psychiatry, University of Cambridge, Herchel Smith Building for Brain and Mind Sciences, Forvie Site, Cambridge Biomedical Campus, Cambridge CB2 0SX, UK.

(Email: pbj21@cam.ac.uk)
}

that have evolved considerably since the evidence was gathered. These studies have indicated a rich landscape of variation in incidence according to standard epidemiological dimensions such as age, sex, social class and ethnicity (McGrath et al. 2004), with further, compound effects visible at the urban neighbourhood level including ethnic density (Kirkbride et al. 2008a). Far less is known about psychosis epidemiology and its public health impact across the full gamut of population settlements, including mixed urban, suburban and rural populations in which the majority of the population lives, a gap that has implications for our 
understanding of causation and for health service provision based on population need.

Nevertheless, major changes have been made to publicly funded mental health services for young adults with first-episode psychotic disorders in the UK, Australia and several European countries. In England, Early Intervention in Psychosis Services (EIS) were introduced in 2002 for young people, aged 14-35 years, presenting to services with symptoms of psychosis who receive a tailored package of care for 3 years before discharge or transfer to appropriate services. Staffing levels were specified on the basis of anticipated incidence rates in the region of 12 to 15 per 100000 person-years (Department of Health, 2001; Lester et al. 2009). The logic for the EI approach included the association between longer duration of untreated psychosis and poorer functional outcome (Marshall et al. 2005), and some evidence from randomized designs that EIS may improve the outcome for young people with psychosis, in terms of fewer relapses, readmissions, symptoms (Craig et al. 2004; Grawe et al. 2006) and cost-effectiveness (Mihalopoulos et al. 2009). However, a Cochrane review on the benefits of EIS concluded that there was insufficient evidence from randomized control trials to draw definitive conclusions as to their effectiveness (Marshall \& Rathbone, 2008). A further follow-up study suggested that any gains were not sustained at 5 years (Bertelsen et al. 2008). A decade since their introduction in England, EIS remain controversial (Marshall \& Rathbone, 2008; Bosanac et al. 2010; Kuehn, 2010; McGorry et al. 2010 ; Pelosi \& Birchwood, 2003) but are the front line for young people who develop psychotic illness.

There have been anecdotal reports of higher than expected caseloads in some, but not all, English EIS. If borne out by epidemiological data, there may be service-based reasons for this, such as the systematic inclusion of 'false-positive' cases boosting caseloads, in addition to the possibility that the original epidemiological predictions (Department of Health, 2001) may have been inadequate, particularly when rates from urban areas were applied indiscriminately to rural settings. These explanations are not mutually exclusive, and can be interrogated using high-quality epidemiological data.

We took the opportunity to estimate the administrative incidence of psychosis and its variation along sociodemographic dimensions using as a case ascertainment system developed in a well-established EIS, CAMEO (www.cameo.nhs.uk), which serves South Cambridgeshire, a mixed urban-rural area of eastern England. Building on our previous epidemiological studies of clinically relevant psychosis, such as the East London First Episode Psychosis (ELFEP) study
(Coid et al. 2008) and the Aetiology and Ethnicity in Schizophrenia and Other Psychoses (AESOP) study (Kirkbride et al. 2006), we designed this EIS on the same epidemiological principles that were used in these studies and the progenitor studies organized by the World Health Organization (WHO; Jablensky \& Sartorius, 2008), so as to facilitate clinical research in a population-based service (Barnett et al. 2007). Similarly, the clinical inclusion criteria were based on clinically relevant psychotic illness rather than the so-called at-risk mental states (ARMS), people with the latter conditions being excluded from the service.

Using data from a 6-year period (2002-2007) we aimed to estimate: (1) the incidence of clinically relevant psychosis in a mixed urban and rural catchment area of the EIS; (2) whether incidence rates were comparable to rates in urban English settings generated by the AESOP and ELFEP studies (conducted prior to the introduction of EIS); and (3) whether rates varied by age, sex and ethnicity as in the urban studies. We hypothesized that age-adjusted rates would be lower than those found in more urban areas of the UK (Kirkbride et al. 2006; Coid et al. 2008) because there is consistent evidence that incidence rates of psychotic disorders are higher in increasingly urban environments (McGrath et al. 2004; March et al. 2008).

\section{Setting}

South Cambridgeshire had an estimated population of 505978 people in 2007 (ONS, 2009a), of whom over $30 \%(n=156058)$ fell within the age range of 17-35 years covered by the CAMEO service. It includes rural areas, small market towns and the university city of Cambridge, where $36 \%$ of the eligible population reside. In terms of ethnicity, the population at risk is predominantly white British (an estimated $76 \%$ in 2007), but with substantial proportions of nonBritish white $(9.7 \%)$, Indian $(2.6 \%)$, Chinese $(2.5 \%)$ and black African (1.4\%) groups. For various reasons, including European Union (EU) expansion in 2004 to include several Eastern European countries, the proportion of the population at-risk from minority ethnic groups has increased from an estimated $20 \%$ in 2002 to $24 \%$ in 2007 . The region is relatively affluent compared with other parts of England; approximately $86 \%$ of our population at-risk (aged 17-35) lived in neighbourhoods less deprived than the median for England in 2007 (Noble et al. 2008). However, substantial pockets of local deprivation exist in the north of the region, including northern parts of Cambridge city and the rural Fenlands. 


\section{Method}

\section{Study design}

We collected data on all people presenting to CAMEO with a potential first episode of psychotic disorder. CAMEO is a National Health Service (NHS)-funded service that offers management for people aged 17-35 years suffering from FEP in Cambridgeshire. The service was commissioned in clearly defined stages, progressively expanding the catchment area in the following way: CAMEO started on 1 January 2002 in Cambridge, South Cambridgeshire, Royston and East Cambridgeshire. The latter two areas left the service on 30 November 2004 due to funding problems but rejoined on 1 June 2007, at which point the service area was also expanded to Huntingdonshire, a district of Cambridgeshire. For the purposes of this study, the cut-off date for inclusion of subjects was 31 December 2007. Data from Peterborough and North Cambridgeshire were not included in the present investigation because the service has been established only recently.

Referrals to the CAMEO service were received from multiple sources including general practitioners (GPs), psychiatric services (secondary care), school and college counsellors, relatives and self-referrals. Efforts were made to promote the service (by raising awareness of psychosis and promoting prompt referral for assessment of suspected cases) throughout the region through ongoing advertising within mental health services and educational lectures, visits to and liaison with GP surgeries, schools and colleges, posting leaflets to all GPs and making introductions during induction courses for mental health staff.

\section{Case ascertainment}

All subjects aged 17-35 years presenting to the CAMEO service with a first episode of psychosis, as defined by the Melbourne criteria of the presence of psychotic symptoms for at least 1 week (McGorry et al. 1996) and duration of antipsychotic treatment of under 6 months at the time of initial assessment, were screened. Referrals were assessed weekly by specialist clinicians, using the semi-structured Positive and Negative Syndrome Scale (PANSS) interview (Kay et al. 1987). All assessments were then discussed with the multidisciplinary team (including at least one or all of the following authors: B.R.L., J.P., G.K.M., E.T.B. or P.B.J.) to ensure that referrals met intake criteria for an ICD-10 psychotic disorder (F10-39), including schizophrenia, bipolar disorders, psychotic depression, schizo-affective disorder, delusional disorder, schizophreniform disorder, substance induced disorders or psychosis not otherwise specified (NOS).
Substance misuse was an exclusion criterion only where psychotic phenomena were clearly and solely present in the context of intoxication. Subjects meeting the inclusion criteria were accepted into the clinical service and so counted in the numerator for the present study. Information on ethnicity was obtained by self-ascription using standard categories. Other demographic data, such as age-at-contact and sex were obtained from subjects during initial assessments.

\section{Population at-risk}

We estimated the denominator population, aged 17-35, in our study areas by using annual mid-term census estimates provided by the Office for National Statistics (ONS) between 2002 and 2007. Mid-year census estimates, stratified by age (yearly), sex and ethnicity, were calculated using annual birth and death rates among different ethnic groups in the UK projected onto the previous year's estimates (or the 2001 Census itself for 2002 estimates) with adjustment made for immigration and emigration (ONS, 2009a). These estimates were not published below local authority level, meaning we had to estimate the yearly population at-risk in one subdistrict of our catchment area (Royston; $n=3629$ ) from the 2001 Census. The estimated denominator data were adjusted to take into account changes in the CAMEO catchment area during the study period.

\section{Statistical analyses}

\section{Variable coding}

We considered all clinically relevant psychotic disorders (F10-39) as the variable defining the numerator that, together with denominator data, was stratified by age (17-19, 20-24, 25-29, 30-35 years), sex, ethnicity and calendar year of inclusion. Because of the low number of minority ethnic groups in our sample, we used four broad ethnicity groupings: white British, non-British white groups, black ethnicities (Caribbean, African and other black groups), and all other ethnicities. We included calendar year as an independent variable to assess and adjust for any changes in incidence (or effectiveness of case finding) over the study period.

To interpret whether incidence rates in our sample were higher than would be expected, we compared our rates with those upon which EIS were predicated (Department of Health, 2001), and also with incidence rates from the two recent observational studies of FEP, mentioned earlier. These covered four urban catchment areas of the UK: East London (the ELFEP study; Coid et al. 2008) and Southeast London, Nottingham 
Table 1. Basic demographic characteristics of sample and crude incidence rates in the CAMEO study

\begin{tabular}{|c|c|c|c|c|}
\hline Denominator population & $\begin{array}{l}\text { Cases } \\
n(\%)\end{array}$ & $\begin{array}{l}\text { Population at-risk } \\
n(\%)\end{array}$ & $\begin{array}{l}\chi^{2} \text { test } \chi^{2}(\mathrm{df}) \\
p \text { value }\end{array}$ & $\begin{array}{l}\text { Crude incidence } \\
\text { rate }(95 \% \mathrm{CI})\end{array}$ \\
\hline Total & $285(100.0)$ & $569921(100.0)$ & & $50.0(44.5-56.2)$ \\
\hline Men & $196(68.8)$ & $296033(51.9)$ & $32.3(1),<0.001$ & $66.2(57.6-76.2)$ \\
\hline Women & $89(31.2)$ & $273888(48.1)$ & & $32.5(26.4-40.0)$ \\
\hline \multicolumn{5}{|l|}{ Age group (years) } \\
\hline $17-19$ & $78(27.4)$ & $87962(15.4)$ & $58.0(3),<0.001$ & $88.7(71.0-110.7)$ \\
\hline $20-24$ & $110(38.6)$ & $170825(30.0)$ & & $64.4(53.4-77.6)$ \\
\hline $25-29$ & $54(18.9)$ & $141182(24.8)$ & & $38.2(29.3-49.9)$ \\
\hline $30-35$ & $43(15.1)$ & $169952(29.8)$ & & $25.3(18.8-34.1)$ \\
\hline \multicolumn{5}{|l|}{ Ethnicity } \\
\hline White British & $206(72.3)$ & $438100(76.9)$ & $5.7(3), 0.13$ & $47.0(41.0-53.9)$ \\
\hline Non-British white & $28(9.8)$ & $56655(9.9)$ & & $49.4(32.8-71.4)$ \\
\hline Black & $11(3.9)$ & $11682(2.0)$ & & $94.2(47.0-168.5)$ \\
\hline Other & $27(9.5)$ & $63484(11.1)$ & & $42.5(28.0-61.9)$ \\
\hline Unknown & $13(4.6)$ & - & - & - \\
\hline
\end{tabular}

df, Degrees of freedom; CI, confidence interval.

and Bristol (the AESOP study; Kirkbride et al. 2006). These studies predated the commissioning of EIS and other functional teams, relying on case ascertainment through general mental health services. Both ELFEP and AESOP calculated age- and sex-standardized rates using direct standardization to the population of England estimated from the 2001 Census. We used the same standard population to calculate standardized rates for our study so as to facilitate comparisons, analyses being restricted to the age range of 18-34 years, common to the three studies (ELFEP, AESOP CAMEO). Given that the incidence of psychosis in the UK has been shown to be elevated in more urban and deprived areas (Kirkbride et al. 2006), we expected age- and sex-standardized rates in South Cambridgeshire to be significantly lower than our reference studies.

\section{Statistical methods}

Incidence per 100000 person-years was calculated with $95 \%$ confidence intervals (CIs). Incidence rate ratios (IRRs) were calculated (with 95\% CIs) using Poisson regression to control for possible confounding. We conducted a sensitivity analysis on subjects with missing ethnicity data by repeating the Poisson regression four times, assuming all such subjects belonged to the white British, non-British white, black and other ethnic groups in turn. The likelihood ratio test (LRT) was applied to assess statistical interactions and model fit. Modelling was conducted in Stata Version 9 (Stata Corporation, USA).

\section{Results}

We identified 294 subjects aged 17-35 years who potentially met inclusion criteria for the study. Five subjects $(1.7 \%)$ did not meet diagnostic criteria for psychosis, and a further four subjects $(1.4 \%)$ had multiple missing data items and were excluded, leaving a sample of 285 from 569921 person-years of follow-up (Table 1). The median age-at-contact in our sample was 22 years for both men and women. People with psychosis tended to be younger than our population at-risk. Men were over-represented among our cases (Table 1), but initial inspection of the data did not suggest differences by ethnicity, although 13 subjects $(4.6 \%)$ were missing ethnicity data (see sensitivity analysis).

\section{Incidence rates of psychosis}

The overall crude incidence in our sample was 50.0 per 100000 person-years (95\% CI 44.5-56.2). Rates were higher for men than women (IRR 2.0, 95\% CI 1.5-2.5), after adjustment for age and ethnicity, but declined for both sexes with increasing age (see Fig. 1). Rates in men were significantly higher than for women until 25-29 years, but we did not observe a statistically significant interaction between age and sex in our model (LRT $p=0.79)$. For both men $(\lambda=124.5,95 \% \mathrm{CI}$ 96.1-161.4) and women $(\lambda=49.8,95 \%$ CI 30.8-76.1), the highest crude incidence of psychosis was in the youngest age group (17-19 years). There was no evidence to suggest the overall incidence of psychosis changed over our 6-year period following adjustment 
Table 2. Incidence rate ratios (IRRs) by ethnicity and sex

\begin{tabular}{|c|c|c|c|c|c|c|}
\hline \multirow[b]{2}{*}{ Ethnic group } & \multicolumn{2}{|l|}{ All cases } & \multicolumn{2}{|l|}{ Men } & \multicolumn{2}{|l|}{ Women } \\
\hline & $n(\%)$ & $\operatorname{IRR}(95 \% \mathrm{CI})^{\mathrm{a}}$ & $n(\%)$ & $\operatorname{IRR}(95 \% \mathrm{CI})^{\mathrm{b}}$ & $n(\%)$ & $\operatorname{IRR}(95 \% \mathrm{CI})^{\mathrm{b}}$ \\
\hline Total & $285(100.0)$ & & $196(100.0)$ & & $89(100.0)$ & \\
\hline White British & $206(72.3)$ & 1.0 & $143(73.0)$ & 1.0 & $63(70.8)$ & 1.0 \\
\hline White non-British & $28(9.8)$ & $1.1(0.8-1.7)$ & $17(8.7)$ & $1.0(0.6-1.7)$ & $11(12.4)$ & $1.4(0.7-2.6)$ \\
\hline Black & $11(3.9)$ & $2.1(1.1-3.8)$ & $6(3.1)$ & $1.7(0.7-3.8)$ & $5(5.6)$ & $2.8(1.1-7.1)$ \\
\hline Mixed and Other & $27(9.5)$ & $0.9(0.6-1.4)$ & $19(9.7)$ & $0.9(0.6-1.5)$ & $8(9.0)$ & $0.8(0.4-1.8)$ \\
\hline Unknown & $13(4.6)$ & & $11(5.6)$ & & $2(2.2)$ & \\
\hline
\end{tabular}

CI, Confidence interval.

a Adjusted for age and sex.

${ }^{\mathrm{b}}$ Adjusted for age.

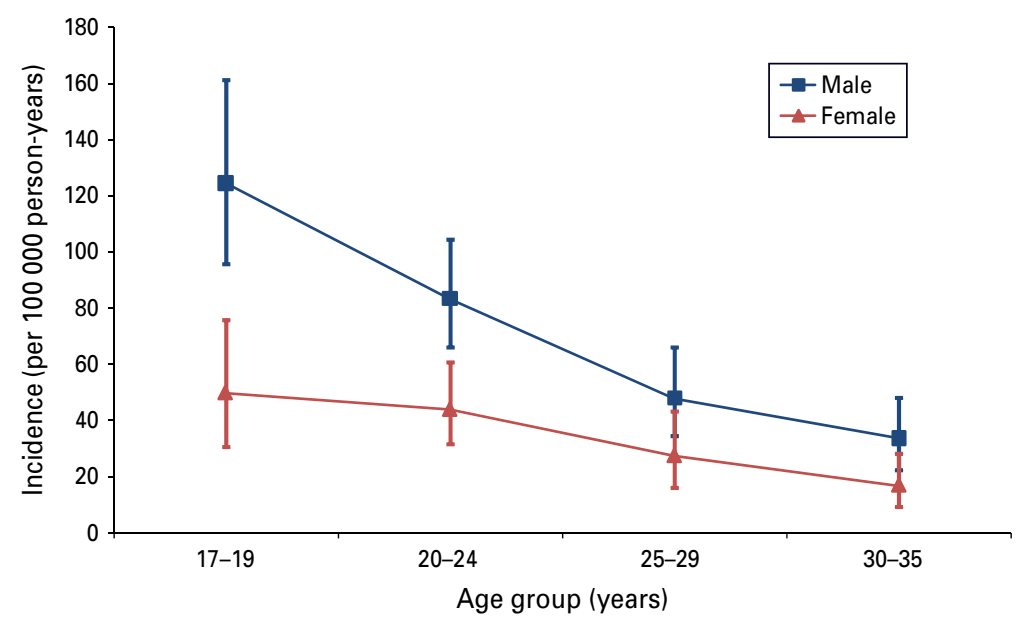

Fig. 1. Crude incidence of all psychotic disorders by age and sex, per 100000 person-years.

for age and sex, or after taking into account possible changes to the denominator population over time (IRR 1.0, 95\% CI 0.9-1.1).

After adjustment for age and sex, the incidence of psychotic disorders was significantly raised among people of black ethnicity (IRR 2.1, 95\% CI 1.1-3.8) compared with the white British group (Table 2), but no other ethnic minority group was observed to have elevated rates of psychosis. We conducted a sensitivity analysis to consider whether subjects with missing data on ethnicity $(n=13)$ could have affected our results. When all subjects with missing ethnicity data were recoded as white British, the raised incidence in the black group persisted (IRR 2.0, $95 \%$ CI 1.1-3.6), after adjustment for age and sex. When we assumed these subjects were from a black ethnic group, the size of this effect increased among men (IRR 4.8, 95\% CI 2.9-8.0) and women (IRR 4.0, $95 \%$ CI 1.8-8.7). Full data are available from the authors.

\section{Comparison with previous English studies of FEP}

Figure 2 shows crude and directly standardized incidence rates of psychosis in our sample compared with those for the same age groups (18-34 years) from the four centres in the AESOP and ELFEP studies. We excluded 37 cases from our sample because they were aged either 17 or $35(n=27 ; 73.0 \%)$ or because data on ethnicity were missing $(n=10 ; 27.0 \%)$, leaving a sample of 248 subjects. The crude and age- and sexstandardized rates in our sample were comparable to those in Nottingham and Bristol but significantly lower than in Southeast and East London (Fig. 2). With additional standardization for ethnicity, the incidence of psychosis became non-significantly different across all catchment areas, suggesting that the excess incidence in London may be attributable to the greater proportion of black and minority ethnic (BME) groups living in the more urban areas. Accordingly, when we repeated this analysis for the white British group only, 


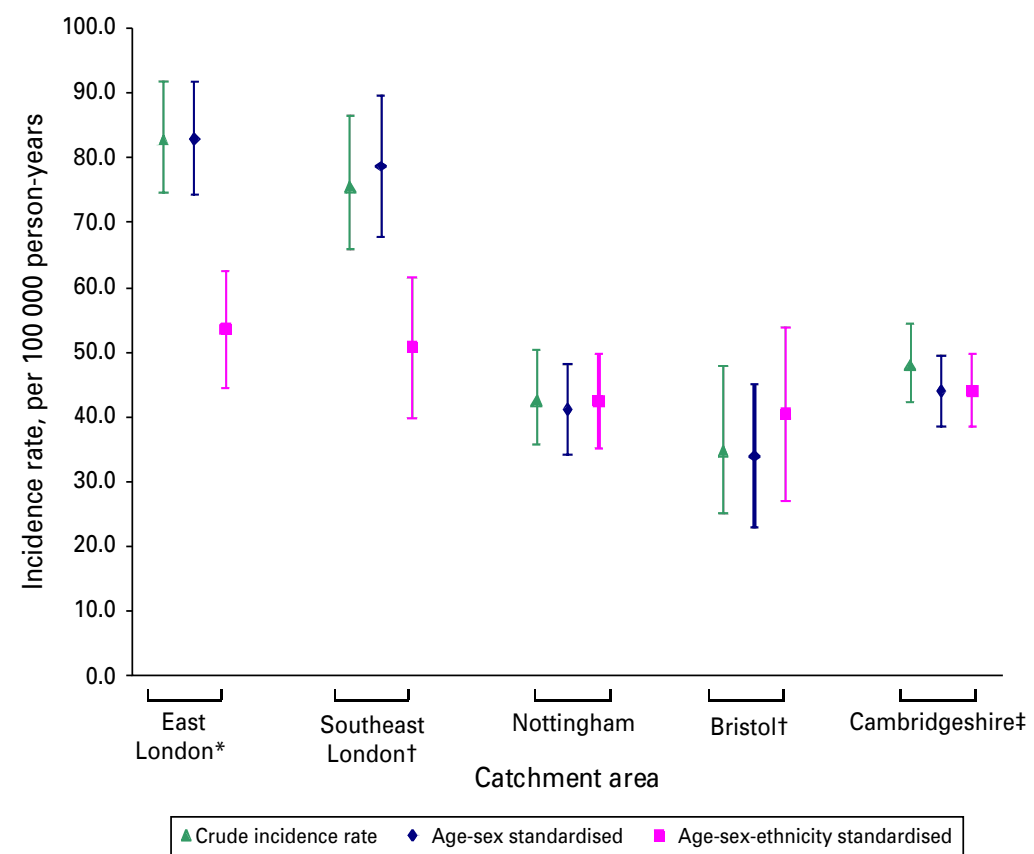

Fig. 2. Comparison of crude and directly standardized incidence rates in Cambridgeshire and four catchment areas of the AESOP and ELFEP studies (directly standardized to the population, aged 18-34 years, of England estimated in the 2001 Census). * Data made available from the authors (Coid et al. 2008). $\uparrow$ Data made available from the authors (Kirkbride et al. 2006).

† Data from the present study.

we found that the crude and age- and sex-standardized incidence rates from the Cambridgeshire EIS were similar to those from the more urban London settings (Fig. 3).

\section{Discussion}

\section{Principal findings}

To our knowledge this is the first epidemiologicallybased study to estimate the incidence of FEP observed through the lens of an EIS, targeting this broad diagnostic group. These administrative rates are higher than originally anticipated when EIS were commissioned in England (Department of Health, 2001), and are similar to those measured by recent observational epidemiological research in substantially more urban settings such as East and Southeast London, Nottingham or Bristol, which predate the introduction of EIS in England. We demonstrated an increased risk of psychosis among people of black ethnicities, after adjusting for sex and age, although this effect was smaller than in other studies (Fearon et al. 2006).

\section{Methodological considerations}

Systematic errors in either our numerator or denominator data could have led us to under- or overestimate our incidence rates, although we went to considerable lengths to minimize such issues. Regarding the numerator, our study identified a clinical sample meeting criteria for FEP detected through an EIS covering a tightly defined epidemiological catchment area over a 6-year period. Cases were ascertained on the basis of diagnoses made using standardized clinical assessments of mental state (PANSS), providing a pragmatic estimate of the psychotic morbidity in our population. We were unable to establish research diagnoses for our sample but all subjects experienced clinically relevant psychotic phenomena (delusion, hallucination, thought disorder or manic syndrome), navigated the referral process and were deemed to be in need of care from secondary mental health services, having met clinical thresholds for specified ICD-10 criteria within the F10-39 range. We did not consider specific psychotic disorders because EIS deliberately avoid diagnostic classification at service entry to accommodate the dynamic phenomenology seen in this setting and to avoid stigma; this is a weakness from the point of view of research and comparability with other studies that could be addressed in future work. However, we can be certain that our results are not due to the inclusion of false-positive cases; that is, people with subclinical ARMS, such as those identified in the general, non-clinical population, who have uncertain predictive value in terms of future psychotic disorder and associated morbidity (Bosanac et al. 2010). Neither funded nor designed to accommodate people with ARMS, our EIS deliberately screened out 


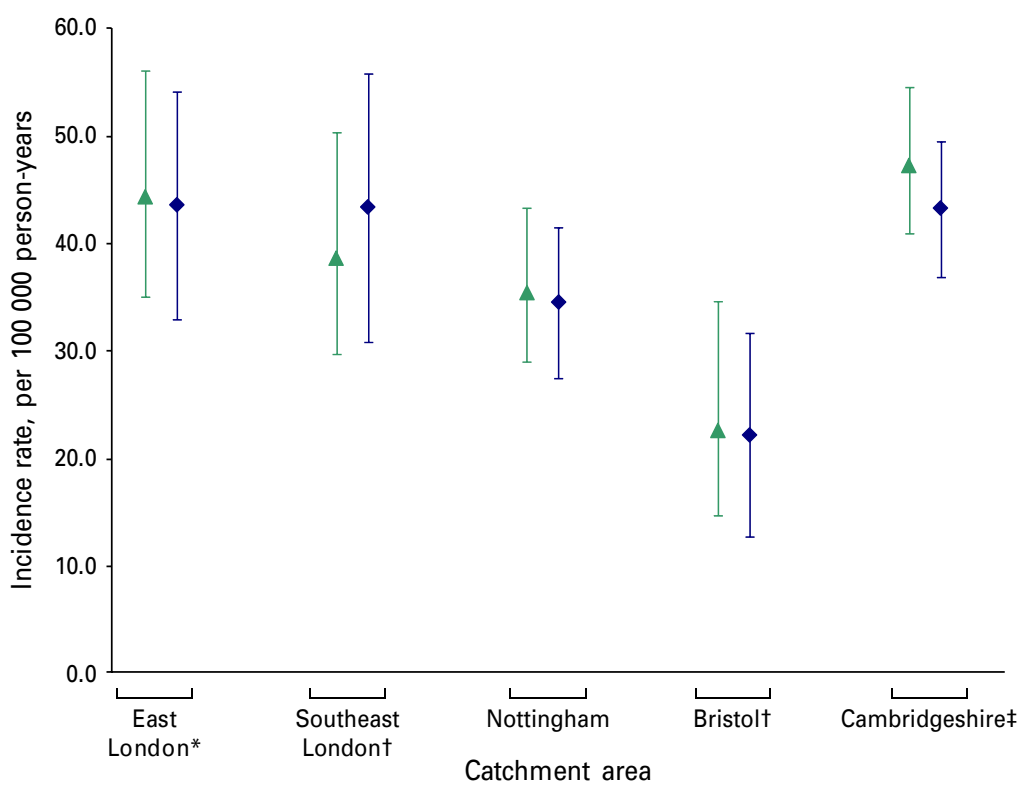

$\Delta$ Crude incidence rate $\bullet$ Age-sex standardised

Fig. 3. Comparison of crude and directly standardized incidence rates for the white British group in Cambridgeshire and four catchment areas of the AESOP and ELFEP studies (directly standardized to the population, aged 18-34 years, of England estimated in the 2001 Census). * Data made available from the authors (Coid et al. 2008). $\uparrow$ Data made available from the authors (Kirkbride et al. 2006). $\$$ Data from the present study.

any such cases, which were subsequently referred to other parts of the health system. Our results are not due to the systematic recruitment of subjects who would have been excluded from previous epidemiological studies, such as those organized by the WHO (Jablensky \& Sartorius, 2008), or our reference cohorts that took the same approach as we did to subjects with psychotic illness in the presence of drug misuse.

We did not conduct a formal leakage study, whereby efforts are made to identify people with FEP missed during the original case ascertainment period, but we made considerable routine and ongoing efforts to ensure that case ascertainment was as complete as possible. The CAMEO early intervention service is one of the longest established and epidemiologically complete EIS in England (Barnett et al. 2005), with plaudits for its quality. As such, it has regular contact with all major service bases in the region and invests considerable time and resources in developing and sustaining contact with primary care, educational establishments and other service bases. There was no evidence that incidence rates increased over the study period, which would otherwise have suggested that the service was not fully optimized at inception. Furthermore, if we had been missing true positive cases our results would be an underestimate of the true effect. The crude incidence of psychotic disorders in our sample was, in fact, higher than would be expected for a less deprived, predominantly rural area, the rates being comparable to those reported previously in more urban settings (Kirkbride et al. 2006). We note that both these potential ascertainment biases (false-positive cases and exclusion of true positives) would tend to negate each other; we have no reason to believe that either was substantial, if present at all, and are confident that they do not explain our findings.

We used annual mid-term census population data to estimate the denominator population. This method more accurately captured changes in the population at-risk over recent years than using data from the 2001 Census, which may have inflated the true incidence rates of psychosis, given an estimated increase in the denominator population between 2001 and 2007 of $6.6 \%$ (from 129390 to 137950) (ONS, 2001, 2009b). These changes was largely driven by net increases in immigration to East Anglia following EU expansion in 2004 (ONS, 2009b). Our mid-term population estimates would have included students in the usual resident population of Cambridge according to census methodology (ONS, 2004), ensuring that we did not underenumerate this important group in the age-at-risk. In general, we adopted an inclusive approach to estimating the denominator, a conservative approach that would tend to underestimate the true incidence of psychotic disorder in our population.

The size of our sample limited our ability to detect differences in incidence rates across ethnic groups. Nevertheless, as in previous research (Fearon et al. 
2006; Kirkbride et al. 2008b), we observed elevated rates of psychosis among people of black ethnicities, effects that persisted when we tested the assumption that people missing ethnicity data were from the white British baseline group. The magnitude of risk in our study was lower than previously estimated for these groups, but we are cautious in our interpretation given the small sample size. Despite our efforts to obtain complete case ascertainment, we cannot exclude the possibility that difficult-to-reach groups that do not fully engage with mental health services may be an explanation for the lower excess risk of psychosis in BME groups in our sample. Self-reported ethnicity is the preferred method of ascribing ethnicity and we have no reason to suspect that this would have led to substantial misclassification, particularly given the relatively homogeneous ethnicity of our study population.

We controlled for age, sex and calendar year in our analyses but acknowledge that other factors, including individual-level socio-economic status or neighbourhood-level socio-economic deprivation, may have confounded our findings and provided additional sources of variation important for health service planning. Our population may differ from other UK settings, but considerable heterogeneity in Cambridgeshire exists with respect to ethnic composition and socio-economic deprivation with some very poor rural communities in the Fenland area. Unfortunately, we did not have access to socioenvironmental data for the present sample but we have established a new survey throughout the Eastern region of England, the Social Epidemiology of Psychoses in East Anglia (SEPEA) study, to address this.

Overall, Cambridgeshire is less deprived, urban and ethnically heterogeneous than many English areas. Based on what is known about the epidemiology of psychosis, it would be reasonable to expect that the incidence of psychotic disorder in our population would be lower than in more urban, deprived populations. Unfortunately, we were unable to compare incidence rates presented here with those in our study population prior to the start of the CAMEO service because no routine incidence data were available at that time. This would represent the gold standard to determine whether EIS does identify excess morbidity, and we acknowledge this limitation. Nevertheless, our results are consistent with this assertion, whether compared with rates anticipated by commissioners or compared with previous empirical observations (Kirkbride et al. 2006; Coid et al. 2008). Our results should therefore be important for health-care planners and commissioners, although further EIS research in larger, urban settings will help to clarify whether other EIS are similarly inundated.

\section{Interpreting our findings}

The fact that the same sociodemographic determinants of incidence, such as age, gender and ethnicity, were apparent in our suburban and rural population as found in more urban settings is of theoretical importance. It indicates that these factors do not account for the 'urbanicity' effect found for schizophrenia and are independent of the determinants of that factor. Ethnicity, in particular, seems to modify risk regardless of urban or rural setting, supporting the notion of the risk being altered by stress-related factors associated with the psychological and cultural environment, such as discrimination, or by other classes of person-environment interaction such as exposure to novel physical toxins, infections or vitamin deficiency (see Kirkbride \& Jones, 2010, for a review). Of note, in other studies high overall incidence rates of psychoses in London seemed to be directly attributable to the greater proportion of BME groups in these areas (Allardyce et al. 2001).

The crude incidence rates presented from this EIS were more than three times higher than anticipated by the original service planning estimates (Department of Health, 2001). It is likely that part of this discrepancy comes from the fact that, hitherto, there has been little evidence on incidence in rural settings compared with urban areas, such that the assumptions about overall rates in the general population have simply been wrong. Further evidence from more inclusive studies will address this. We have argued that our relatively high rates are not due to the inclusion of false-positive cases and ARMS. It is feasible that, set up as specialist teams, EIS are particularly effective in eliciting referrals of true positives and engaging them long enough for assessments to be made. That said, the fact that we did not have a formal leakage study, as was undertaken in our comparison samples, suggests to us that those studies and general mental health services did not massively underestimate morbidity. Another possible reason for more rural areas to look like cities could be the uniformly high prevalence of cannabis use by young people in the UK. The association between cannabis and psychosis incidence is certainly complex (Moore et al. 2007), and analysis of secular trends that are relatively static over recent years (Frisher et al. 2009) does not support the notion that the saturation of rural areas and also urban areas has led the former to behave more like the latter in terms of these illnesses.

The most obvious reason for the discrepancy between our data and the figures for EIS planning used in England (around 15 per 100000 person-years) is that the latter are predicated largely on the incidence of schizophrenia whereas we know that only around 
one-third of first-onset psychotic illness is classified as such at first presentation (Kirkbride et al. 2006), although there is a net evolution towards that diagnostic category over the first 3 years and beyond (Amin et al. 1999). Furthermore, the incidence of psychosis is higher in young adults than in the population as a whole, and EIS are targeted at the former group.

We are left with the conclusions that the incidence of psychotic illness in our mixed urban-rural catchment is fairly similar, on average, to highly urban cities, and that there is variation within all settings according to sociodemographic variables. Ethnic origin from a visibly different migrant community is a potent indicator of risk regardless of crude population setting but is influenced by factors more proximal to the individual, such as ethnic density and assimilation (Kirkbride et al. 2008a). Combined with other factors, not least those genetic and environmental entities that are associated with sociodemographic characteristics and that, themselves, modify risk, these data are further evidence of the complex eco-epidemiology of psychosis (March et al. 2008). We know that any urban-rural effect on administrative incidence is likely to be non-linear (Croudace et al. 2000), with pockets of extremely high incidence of non-affective psychosis in some neighbourhoods juxtaposed with average areas. There needs to be a high degree of granularity in any picture of the occurrence of psychosis, whether this concerns causation in a bio-psycho-social model or the health needs of the population used to plan services. Regardless of whether the field decides that functional specialization in mental health services in general, and in EIS in particular, has value and should be preserved, we urge those who make decisions about the mental health needs of populations to be aware of the devil in the detail.

\section{Acknowledgements}

The CAMEO service provided by the Cambridgeshire and Peterborough Foundation Trust is funded by the Cambridgeshire Primary Care Trust. CAMEO hosts research funded by the National Institute for Health Research (NIHR programme grant RP-PG-0606-1335), the Wellcome Trust and the Medical Research Council (MRC), and has previously hosted research funded by the Stanley Medical Research Institute and GlaxoSmithKline. J.B.K. was supported by a Sir Henry Wellcome Research Fellowship from the Wellcome Trust (grant code: WT085540 for SEPEA).

\section{Declaration of Interest}

E.T.B. is employed half-time by the University of Cambridge and half-time by GlaxoSmithKline (GSK), and he holds shares in GSK and the Brain Resource Company. P.B.J. directs the NIHR CLAHRC for Cambridgeshire and Peterborough.

\section{References}

Allardyce J, Boydell J, Van Os J, Morrison G, Castle D, Murray RM, McCreadie RG (2001). Comparison of the incidence of schizophrenia in rural Dumfries and Galloway and urban Camberwell. British Journal of Psychiatry 179, 335-339.

Amin S, Singh SP, Brewin J, Jones PB, Medley I, Harrison G (1999). Diagnostic stability of first-episode psychosis. Comparison of ICD-10 and DSM-III-R systems. British Journal of Psychiatry 175, 537-543.

Barnett JH, Sahakian BJ, Werners U, Hill KE, Brazil R, Gallagher O, Bullmore ET, Jones PB (2005). Visuospatial learning and executive function are independently impaired in first-episode psychosis. Psychological Medicine 35, 1031-1041.

Barnett JH, Werners U, Secher SM, Hill KE, Brazil R, Masson K, Pernet DE, Kirkbride JB, Murray GK, Bullmore ET, Jones PB (2007). Substance use in a population-based clinic sample of people with first-episode psychosis. British Journal of Psychiatry 190, 515-520.

Bertelsen M, Jeppesen P, Petersen L, Thorup A, Ohlenschlaeger J, le Quach P, Christensen TO, Krarup G, Jorgensen P, Nordentoft M (2008). Five-year follow-up of a randomized multicenter trial of intensive early intervention vs standard treatment for patients with a first episode of psychotic illness: the OPUS trial. Archives of General Psychiatry 65, 762-771.

Bosanac P, Patton GC, Castle DJ (2010). Early intervention in psychotic disorders: faith before facts? Psychological Medicine 40, 353-358.

Coid JW, Kirkbride JB, Barker D, Cowden F, Stamps R, Yang M, Jones PB (2008). Raised incidence rates of all psychoses among migrant groups: findings from the East London first episode psychosis study. Archives of General Psychiatry 65, 1250-1258.

Craig TKJ, Garety P, Power P, Rahaman N, Colbert S, Fornells-Ambrojo M, Dunn G (2004). The Lambeth Early Onset (LEO) Team: randomised controlled trial of the effectiveness of specialised care for early psychosis. British Medical Journal 329, 1067-1060.

Croudace TJ, Kayne R, Jones PB, Harrison GL (2000). Non-linear relationship between an index of social deprivation, psychiatric admission prevalence and the incidence of psychosis. Psychological Medicine 30, 177-185.

Department of Health (2001). Mental Health Policy Implementation Guide. National Health Service: London

Fearon P, Kirkbride JB, Morgan C, Dazzan P, Morgan K, Lloyd T, Hutchinson G, Tarrant J, Lun Alan Fung W, Holloway J, Mallett R, Harrison G, Leff J, Jones PB, Murray RM (2006). Incidence of schizophrenia and other psychoses in ethnic minority groups: results from the MRC AESOP Study. Psychological Medicine 36, 1541-1550. 
Frisher M, Crome I, Martino O, Croft P (2009). Assessing the impact of cannabis use on trends in diagnosed schizophrenia in the United Kingdom from 1996 to 2005. Schizophrenia Research 113, 123-128.

Grawe RW, Falloon IRH, Widen JH, Skogvoll E (2006). Two years of continued early treatment for recent-onset schizophrenia: a randomised controlled study. Acta Psychiatrica Scandinavica 114, 328-336.

Jablensky A, Sartorius N (2008). What did the WHO studies really find? Schizophrenia Bulletin 34, 253-255.

Kay SR, Fiszbein A, Opler LA (1987). The positive and negative syndrome scale (PANSS) for schizophrenia. Schizophrenia Bulletin 13, 261-276.

Kirkbride JB, Boydell J, Ploubidis GB, Morgan C, Dazzan P, McKenzie K, Murray RM, Jones PB (2008a). Testing the association between the incidence of schizophrenia and social capital in an urban area. Psychological Medicine 38, 1083-1094.

Kirkbride JB, Coid JW, Barker D, Cowden F, Stamps R, Yang M, Jones PB (2008b). Psychoses, ethnicity and socio-economic status. British Journal of Psychiatry 193, $18-24$.

Kirkbride JB, Fearon P, Morgan C, Dazzan P, Morgan K, Tarrant J, Lloyd T, Holloway J, Hutchinson G, Leff JP, Mallett RM, Harrison GL, Murray RM, Jones PB (2006). Heterogeneity in incidence rates of schizophrenia and other psychotic syndromes: findings from the 3-center AESOP study. Archives of General Psychiatry 63, 250-258.

Kirkbride JB, Jones PB (2010). The prevention of schizophrenia - what can we learn from ecoepidemiology? Schizophrenia Bulletin. Published online: 25 October 2010. doi:10.1093/schbul/sbq120.

Kuehn BM (2010). Early interventions for schizophrenia aim to improve treatment outcomes. Journal of the American Medical Association 304, 139-145.

Lester H, Birchwood M, Bryan S, England E, Rogers H, Sirvastava N (2009). Development and implementation of early intervention services for young people with psychosis: case study. British Journal of Psychiatry 194, 446-450.

March D, Hatch SL, Morgan C, Kirkbride JB, Bresnahan M, Fearon P, Susser E (2008). Psychosis and place. Epidemiologic Reviews 30, 84-100.

Marshall M, Lewis S, Lockwood A, Drake R, Jones P, Croudace T (2005). Association between duration of untreated psychosis and outcome in cohorts of first-episode patients: a systematic review. Archives of General Psychiatry 62, 975-983.

Marshall M, Rathbone J (2008). Early intervention for psychosis. Cochrane Database of Systematic Reviews, 18, CD004718.

McGorry P, Johanessen JO, Lewis S, Birchwood M, Malla A, Nordentoft M, Addington J, Yung A (2010). Early intervention in psychosis: keeping faith with evidence-based health care. Psychological Medicine 40, 399-404.

McGorry PD, Edwards J, Mihalopoulos C, Harrigan SM, Jackson HJ (1996). EPPIC: an evolving system of early detection and optimal management. Schizophrenia Bulletin 22, 305-326.

McGrath J, Saha S, Welham J, El Saadi O, MacCauley C, Chant D (2004). A systematic review of the incidence of schizophrenia: the distribution of rates and the influence of sex, urbanicity, migrant status and methodology. BMC Medicine 2, 13.

Mihalopoulos C, Harris M, Henry L, Harrigan S, McGorry P (2009). Is early intervention in psychosis cost-effective over the long term? Schizophrenia Bulletin 35, 909-918.

Moore THM, Zammit S, Lingford-Hughes A, Barnes TRE, Jones PB, Burke M, Lewis G (2007). Cannabis use and risk of psychotic or affective mental health outcomes: a systematic review. Lancet 370, 319-328.

Noble M, McLennan D, Wilkinson K, Whitworth A, Barnes H, Dibben C (2008). The English Indices of Deprivation 2007. Communities and Local Government: London.

ONS (2001). Census 2001. Office for National Statistics (http://www.ons.gov.uk/census/get-data/index.html). Accessed 29 December 2009.

ONS (2004). Census 2001 Definitions. The Stationery Office: London.

ONS (2009a). Population Estimates by Ethnic Group Methodology Paper. Office for National Statistics: Newport.

ONS (2009b). Table 8 - Mid-2007 UK, England and Wales, Scotland and Northern Ireland population estimates. In Population Estimates for UK, England and Wales, Scotland and Northern Ireland - Current Datasets. Office for National Statistics: Fareham.

Pelosi AJ, Birchwood M (2003). Is early intervention for psychosis a waste of valuable resources? British Journal of Psychiatry 182, 196-198. 